\title{
RIGIDITY AMONG PRIME-KNOT COMPLEMENTS
}

\author{
BY WILBUR WHITTEN ${ }^{1}$
}

An unpublished result of Hempel and Waldhausen states that the group of a prime knot in $S^{3}$ determines the type of the knot provided that each nontrivial (tame) knot $K$ satisfies the unique imbedding property (UIP), that is, if any imbedding $E(K) \rightarrow S^{3}$ of the exterior of $K$ into $S^{3}$ extends to an autohomeomorphism of $S^{3}$. Since we do not yet know that all nontrivial knots have the UIP, much less property $P$, this result suggests four old questions.

(1) Does the group of a prime knot determine the complement?

(2) Does the group of a prime knot determine the type of the knot?

(3) Do knot complements determine knot types?

(4) Do all nontrivial knots satisfy the UIP?

Partial answers abound - see, for example, Simon's remarks in [K, Problem 1.13, p. 278] and the extensive comments of Gordon in [G] for backgroundbut these partial results do not resolve any of these questions. The principal announcement in this paper is that the answer to Question (1) is affirmative.

RIGIDITY THEOREM. Prime knots $\left(\subset S^{3}\right)$ with isomorphic groups have homeomorphic complements.

REMARK. Since the group of a prime knot cannot be isomorphic to that of a composite knot [FW, Lemma 2, p. 1286], the Rigidity Theorem answers Question (1) affirmatively.

The Rigidity Theorem follows from Proposition 1 and recent (combined) work of Culler, Gordon, Luecke, and Shalen ([CGLS ${ }_{1}$, Corollary 2, p. 43] or [CGLS ${ }_{2}$, Corollary 2]). Let $Q$ denote the rationals, let $r \in Q \cup\{\infty\}$, and let $K(r)$ denote the closed, orientable 3-manifold obtained by $r$-surgery on a tame knot $K \subset S^{3}$.

PROPOSITION 1. If there exist prime knots with isomorphic groups and nonhomeomorphic complements, then there exist a nontrivial knot $K$ and an integer $m$ such that

(1) $K(1 / m) \cong S^{3}$, and

(2) $|m| \neq 0,1$, or 2 .

OUTLINE OF PROOF. Let $J_{1}$ and $J_{2}$ be prime knots with isomorphic groups and nonhomeomorphic complements. Then, as is well known, $J_{i}$ is a cable knot, $J\left(p_{i}, q_{i}, K_{i}\right)$, about a nontrivial knot $K_{i}(i=1,2)$ (see, for example, [K, Problem 1.13, p. 278]), and so there exist annuli, $A_{1}$ and $A_{2}$,

Received by the editors March 21, 1985 and, in revised form, December 18, 1985.

1980 Mathematics Subject Classification (1985 Revision). Primary 57M25, 57R65.

${ }^{1}$ I wish to thank the Institute for Advanced Study, Princeton, New Jersey, for its hospitality while part of this work was done. The ONR grant N 0014-85-K-0099 provided partial support. 
and solid tori, $V_{1}$ and $V_{2}$, such that $E\left(J_{i}\right) \cong E\left(K_{i}\right) \bigcup_{A_{i}} V_{i}(i=1,2)$. By an application of Johannson's deformation theorem [Ja, Theorem X.21, p. 212], we can find a homeomorphism $f: E\left(K_{1}\right) \rightarrow E\left(K_{2}\right)$ such that $f\left(A_{1}\right)=A_{2}$. Hence, if we orient $K_{i}$ and let $\left(\mu_{i}, \lambda_{i}\right)$ be a (standard) meridian-longitude pair on $\partial E\left(K_{i}\right)$, then $f$ takes a $\left(p_{1}, q_{1}\right)$-curve on $\partial E\left(K_{1}\right)$ to a $\pm\left(p_{2}, q_{2}\right)$-curve on $\partial E\left(K_{2}\right)$. For homological reasons, we have $\left|p_{1}\right|=\left|p_{2}\right|$ and $\left|q_{1}\right|=\left|q_{2}\right|$. Changing orientations, if need be, we can guarantee that $q_{1}=q_{2}=q \geq 2$; set $p_{1}=p$, and note that $p_{2}=\varepsilon p$, with $\varepsilon \in\{-1,1\}$.

Homologically, $f_{*}\left(\lambda_{1}\right)= \pm \lambda_{2}$, and $f_{*}\left(\mu_{1}\right)= \pm \mu_{2}+m \lambda_{2}$ (for some $m \in Z$ ); also, $f_{*}\left(p \mu_{1}+q \lambda_{1}\right)= \pm\left(\varepsilon p \mu_{2}+q \lambda_{2}\right)$. It follows easily that $m p= \pm 2 q$. Hence $1 \leq|p| \leq 2$ and

$$
|m|= \begin{cases}2 q, & \text { if }|p|=1 \\ q(\text { odd }), & \text { if }|p|=2\end{cases}
$$

Therefore, $|m| \neq 0,1$, or 2 , since $q \geq 2$. Since $f_{*}\left(\mu_{1}\right)= \pm \mu_{2}+m \lambda_{2}$, either $K_{2}(1 / m) \cong\left(S^{3}, K_{1}\right)$ or $K_{2}(-1 / m) \cong\left(S^{3}, K_{1}\right)$.

COROLLARY 2. There exist at most two distinct prime knots with a given group.

PROOF. Let $\left\{K_{1}, K_{2}, \ldots\right\}$ be any collection of prime knots with $\pi_{1} E\left(K_{i}\right) \approx$ $\pi_{1} E\left(K_{j}\right)$, for all $i$ and $j$. By the Rigidity Theorem, we have $E\left(K_{i}\right) \cong E\left(K_{j}\right)$, for all $i$ and $j$. By [CGLS ${ }_{1}$, Corollary 3, p. 43] or by $\left[\mathbf{C G L S}_{2}\right.$, Corollary $3]$, the collection $\left\{K_{1}, K_{2}, \ldots\right\}$ contains representatives from, at most, two distinct knot types.

Complete proofs and other results will appear in $[\mathbf{W}]$. I wish to thank M. Boileau, F. González-Acuña, C. Gordon, K. Murasugi, and J. Simon for helpful comments.

\section{REFERENCES}

[CGLS 1 ] M. Culler, C. Gordon, J. Luecke, and P. B. Shalen, Dehn surgery on knots, Bull. Amer. Math. Soc. (N.S.) 13 (1985), 43-45.

[CGLS $\mathbf{C G}_{2}$, Dehn surgery on knots, MSRI preprint.

[FW] C. D. Feustel and W. Whitten, Groups and complements of knots, Canad. J. Math. 30 (1978), 1284-1295.

[G] C. McA. Gordon, Some aspects of classical knot theory, Knot Theory (Proc., Plans-sur-Bex, Switzerland, 1977), Lecture Notes in Math., vol. 685, Springer-Verlag, Berlin and New York, 1978, pp. 1-60.

[Ja] W. Jaco, Lectures on three-manifold topology, CBMS Regional Conf. Ser. in Math., no. 43, Amer. Math. Soc., Providence, R. I., 1980.

[K] R. Kirby, Problems in low-dimensional manifold theory, Proc. Sympos. Pure Math., vol. 32, Amer. Math. Soc., Providence, R. I., 1978, pp. 273-312.

[W] W. Whitten, Knot complements and groups, Topology (to appear).

DEPARTMENT OF MATHEMATICS, UNIVERSity OF SOUTHWESTERN LOUISIANA, LAFAYETTE, LOUISIANA 70504 (Current address)

Department of MATHEMATICS, University of IOWA, IOWA City, IOWA 52242 